УДК. 004.052

М.С. Солодовник

Одеська національна академія харчових технологій, вул. Дворянська, 1/3, Одеса, 65026

\title{
ЗАСТОСУВАННЯ НЕЙРО-НЕЧІТКОГО ПІДХОДУ ДЛЯ ПІДВИЩЕННЯ НАДІЙНОСТІ І ОП- ТИМАЛЬНОЇ РОБОТИ КОМП'ЮТЕРНОЇ МЕРЕЖІ
}

У статті представлений аналіз існуючих традиційних методів пошуку оптимального маршруту при маршрутизації в корпоративних мережах. В якості альтернативи традиційним методам, запропонований нейро-нечіткий підхід для оптимізачії процесу мармрутизації з урахуванням прогнозування виходу з ладу жорсткого диска сервера. Для прогнозування використовувався пакет FuzzyTech середовища Matlab. Написана програма, яка базується на апараті нейро-нечіткої логіки. В якості вхідних даних взято дані корпорації Google , які були опубліковані на конференції в Торонто. На підставі ичих даних, спрогнозована ймовірність відмови жорсткого диска сервера , щзо призведе до змін в архітектурі корпоративної мережі, а, отже, принесе зміни $і$ в процес марирутизаиії. Обговорено переваги нейро-нечіткого методу перед традиційними, а саме врахування думки експерта , здатність до самонавчання $i$ здатність працювати з нелінійними функціями.

Ключові слова: нейро-нечітка мережа, адаптивна маршрутизачія, інтелектуальне прогнозування, нейронна мережа, навчання мережі, матричний алгоритм, самонавчання мережі, правила навчання, функиія активації, гібридна мережа, оптимальний маршрут.

\section{М.С. Солодовник}

Одесская национальная академия пищевых технологий, ул. Дворянская, 1/3, Одесса, 65026

\section{ПРИМЕНЕНИЕ НЕЙРО-НЕЧЕТКОГО ПОДХОДА ДЛЯ ПОВЫШЕНИЯ НАДЕЖНОСТИ ОП- ТИМАЛЬНОЙ РАБОТЫ КОМПЬЮТЕРНОЙ СЕТИ}

В статье представлен анализ существующих традищионных методов поиска оптимального маршрута при маршрутизаџии в корпоративных сетях. В качестве альтернативы традиционному методу, предложен нейро-нечеткий подход для оптимизаџии процесса маршрутизаџии с учетом прогнозирования выхода из строя жёсткого диска сервера. Для прогнозирования использовался пакет FuzzуTесh среды Matlab. Написана программа, которая основана на аппарате нейронечеткой логики. В качестве исходных данных взяты данные корпорации Goоgle, которые были опубликованные на конференичи в Торонто. На основании этих данных, спрогнозирована вероятность отказа жесткого диска сервера, что приведет к изменениям в архитектуре корпоративной сети, а, следовательно, принесет изменения и в процесс мармрутизации. Оговорены преимущества нейро-нечеткого метода перед традиционными, а именно учет мнения эксперта, способность к самообучению и способность работать с нелинейными функциями.

Ключевые слова: нейро-нечеткая сеть, адаптивная маршрутизация, интеллектуальное прогнозирование, нейронная сеть, обучение сети, матричный алгоритм, самообучение сети, правила обучения, функция активации, гибридная сеть, оптимальный мармрут.

\author{
M.S. Solodovnik
}

Odessa national academy of food technologies, 1/3 Dvorjanskaja st., Odessa, 65026

\section{APPLICATION OF NEURO-FUZZY APPROACH TO IMPROVE RELIABILITY AND OPTIMAL WORK OF COMPUTER NETWORK}

The paper presents analysis of the existing traditional methods of finding the best route for routing in networks. As an alternative to the traditional method, was proposed a neuro-fuzzy approach for the optimization of the routing process with the prediction of failure of the server's hard disk drive. For the prediction we used the package FuzzyTech environment Matlab. The program that is based on the unit neuro-fuzzy logic was written. The initial data are taken data corporation Google, which was published at a conference in Toronto. Based on these data, the predicted probability of failure of the hard disk server, resulting in changes in the architecture of a corporate network, and consequently bring changes in the routing process. Specific advantages of the neuro-fuzzy method over the traditional, namely, accounting expert opinion, the ability to self-learning and the ability to work with non-linear functions.

Keywords: neuro-fuzzy network, adaptive routing, intelligent prediction, neural network, training of the network, matrix algorithm, self-learning network, learning rules, activation function, hybrid network, optimal route. 


\section{I. ВВЕДЕНИЕ}

Высокие требования, предъявляемые к эффективности, надёжности и скорости передачи данных в компьютерных сетях (КС) способствуют появлению новых методов и алгоритмов маршрутизации и надежности сетевого оборудования. Для своевременной и безопасной доставки информации в КС важную роль играет оптимальная маршрутизация, стойкая к различным отказам, эффективная в условиях повышенной нагрузки сети, и предотвращающая несанкционированный доступ к информации [11].

В процессе обслуживания корпоративной сети следует учитывать её модификации в будущем, перепланировку, внедрение новых узлов или линий связи, возможные отказы серверов, т.е. надежность сети. При этом возникает необходимость изменения таблицы маршрутизации за кратчайшие сроки, т.е. адаптировать маршрутизатор. Этим обуславливается разработка наиболее эффективных алгоритмов поиска оптимальных маршрутов от узла к узлу учитывающих возможные изменения КС.

Вопросу разработки методов повышения эффективности маршрутизации, и надежности сетевого оборудования посвящены работы таких ученых, как Д. В. Куракин, О. Я. Кравец, И. П. Норенков, А. Леинванд, Б. Пински, Б. С. Гольдштейн и т.д. $[1,4,5,7]$. Однако, несмотря на широкий спектр исследований в данной области, следует отметить, что в проанализированных работах остаются открытыми научные задачи использования самообучающихся адаптивных алгоритмов маршрутизации или интеллектуальных технологий. Также в публикациях отсутствуют исследования связанные с изменением маршрутов КС на основе прогнозной информации о возможном выходе из строя компьютерного оборудования. Следует отметить, что в последнее время интеллектуальные методы [9, 12, 13] получают широкое развитие в компьютерных системах (КС) и рассмотрение данного научного подхода применительно к алгоритмам маршрутизации с учетом информации о надежности элементов КС является актуальным [14].

Предлагаемая работа посвящена вопросу повышения эффективности работы КС на основе адаптивной (нейро-нечеткой) маршрутизации учитывающей показатели надежности структурных элементов КС.

Целью работы является исследование на практике нового подхода (интеллектуального) поиска кратчайшего маршрута при адаптивной маршрутизации, который будет учитывать мнение эксперта - системного администратора и иметь способность самообучаться на основе обучающей выборки. А также учитывать вероятность выхода из строя элементов КС, на примере жесткого диска сервера.

\section{II. ПРОГНОЗИРОВАНИЕ ВЫХОДА ИЗ СТРОЯ ЖЁСТКОГО ДИСКА КОРПОРАТИВ- НОЙ СЕТИ. ОСНОВНЫЕ ПОЛОЖЕНИЯ}

Для начала авторами рассмотрен вопрос прогнозирования выхода из строя жесткого диска. В качестве входных данных для прогнозирования взята статистическая информация, предоставленная корпорацией Google Inc. Эти данные впервые были представлены для всеобщего обозрения в 2007 году на конференции USENIX с подробной статистикой о том, как и почему в компании Google выходят из строя жесткие диски (рисунок 1).

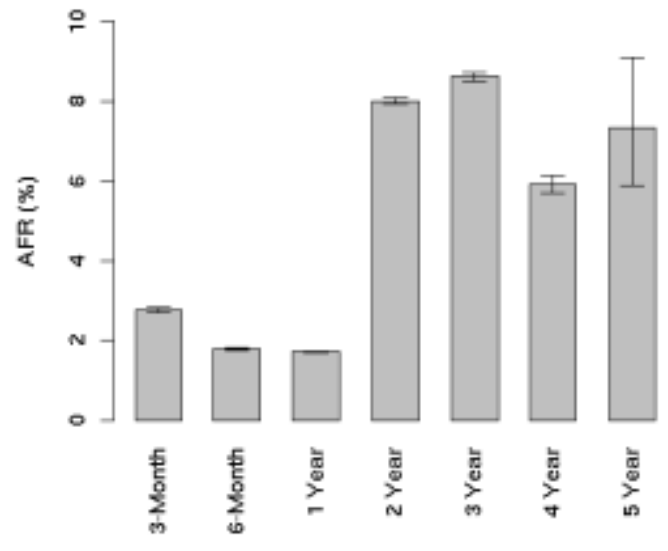

Рисунок 1 - Зависимость выхода из строя жестких дисков от времени эксплуатациии

В данном графике учитывались условия эксплуатации жестких дисков, а именно частота обращения к данному диску и его температура (вероятность поломки диска возрастает, если эксплуатировать его при температуре ниже $35^{\circ} \mathrm{C}$, а особенно - ниже $25^{\circ} \mathrm{C}$ ). Основная статистическая информация, связанная с результатами измерений (среднеквадратичное отклонение, математическое ожидание и т.д. представлена на сайте компании) $[9,10]$. Для удобства оперирования данными данная диаграмма была преобразована в график с линейной зависимостью $(\underline{t}-$ время, месяц, $n-$ количество, штук) (рисунок 2).

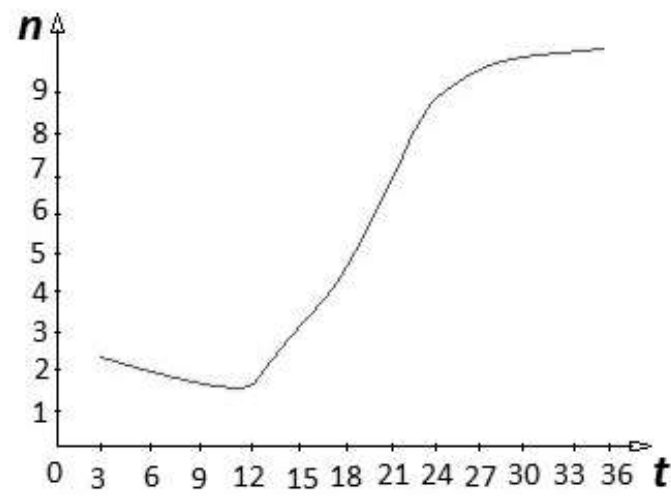

Рисунок 2 - График зависимости выхода из строя жестких дисков от времени эксплуатации 
На основании этих данных, авторами спрогнозирована вероятность выхода из строя жесткого диска на дальнейший временной период. С помощью аппарата нейро-нечеткой логики (ANFIS) предсказывается вероятность возникновения критической ошибки. Также можно прогнозировать выход из строя и других элементов КС (маршрутизаторов, коммутаторов, мостов и т.д.) Таким образом, учитывая эту информацию, маршрутизатор сможет заранее выбрать другой (оптимальный) маршрут.

Рассмотрим детальнее метод прогнозирования вероятности отказа с помощью аппарата нейро-нечеткой логики.

Предположим, что нечеткая модель гибридной сети будет иметь 4 переменные. При этом первая переменная будет соответствовать вероятности возникновения ошибки на текущий день, вторая - вероятности возникновения ошибки на текущий день, вторая - вероятности возникновения ошибки в предыдущий день, т. е. на день $(i-$ $1)$, где $i$ - текущий день. Тогда третья входящая переменная будет соответствовать вероятности возникновения ошибки на $(i-2)$ день, а четвертая на $(i-3)$ день эксплуатации.

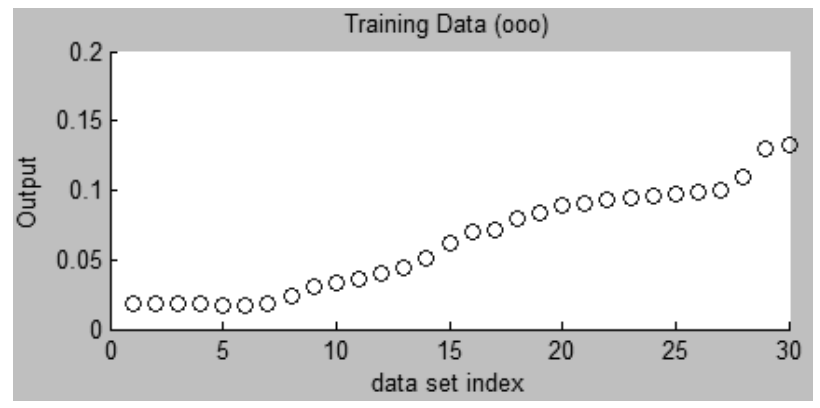

Рисунок 3 - Графический интерфейс редактора ANFIS после загрузки в него обучающих данных

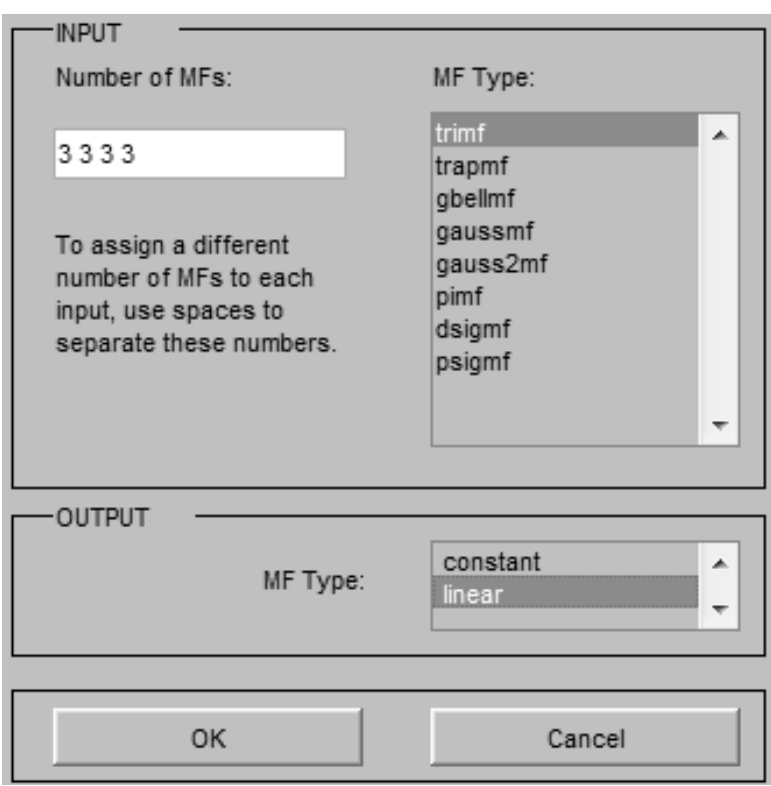

Рисунок 4 - Диалоговое окно для задания количества и типа функции принадлежности
Эти обучающие данные были сведены в соответствующую таблицу. Для удобства, обучающую выборку сохраним во внешнем файле HDD1.dat. После этого воспользуемся редактором ANFIS, в который и загрузим этот файл.

Вызов редактора осуществляется командой anfisedit из командной строки среды MATLAB. Перед генерацией структуры системы нечеткого вывода зададим основные параметры данной структуры: для каждой входной переменной по 3 лингвистических терма, в качестве типа функций их принадлежности - треугольные функции.

Графический вид будет представлен в виде линейной зависимости и будет иметь вид, приведенный на рисунке 3.

Далее автором была сгенерирована система FIS (кнопка Generate FIS) с соответствующими параметрами функции принадлежности (рисунок 4).

Далее был проведен процесс обучения построенной гибридной сети. В качестве метода обучения был выбран гибридный метод с уровнем ошибки равным нулю, и количеством циклов обучения равным тридцати. Результат процесса обучения представлен на рисунке $5[3,8]$.

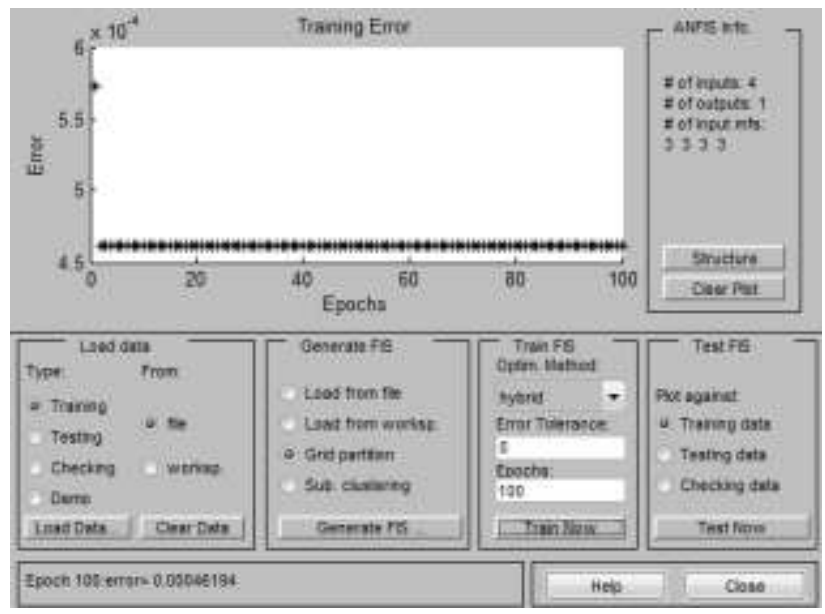

Рисунок 5 - График зависимости ошибок обучения от количества цииклов обучения

Проанализировав данный график, можно сделать вывод, что процесс обучения практически заканчивается после 2-го цикла. Это значит, что полученная FIS-система имеет высокую степень обучаемости.

Структура системы будем иметь вид, приведенный на рисунке 6.

Далее была выполнена проверка полученной системы на адекватность. Для этого был проведён ретроспективный прогноз значения вероятности отказа жёсткого диска сервера на следующий день. Полученное значение было сравнено с графическим значением (рисунок 2). Для прогноза использовалась функция evalfis из командной строки редактора. В качестве аргумента данной функции автор указал вектор значений вероятности возникновения отказа жесткого диска сервера на текущий 


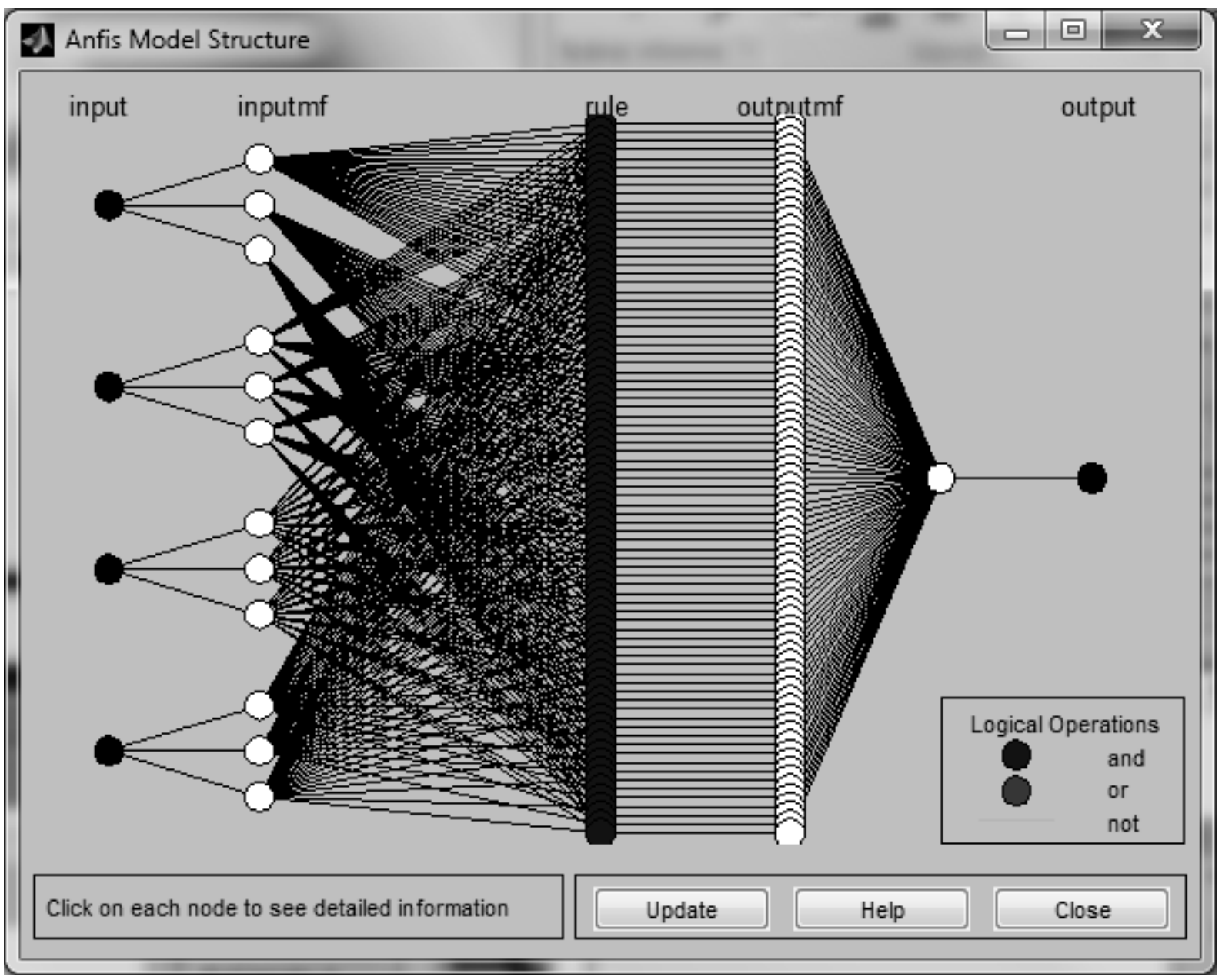

Рисунок 6 - Структура сгенерированной системы нечеткого вывода (нейро-нечеткая сеть)

\begin{tabular}{|c|c|c|c|c|}
\hline \multicolumn{5}{|c|}{ Anfis Editor: Untitled } \\
\hline \multirow[t]{2}{*}{ File } & Edit $V_{i}$ & View & \multirow{2}{*}{\multicolumn{2}{|c|}{ Training Data (000) }} \\
\hline & New FIS... & - & & \\
\hline & Import & $\rightarrow$ & \multirow{2}{*}{\multicolumn{2}{|c|}{$\begin{array}{l}\text { From Workspace... } \\
\text { From File... } \quad \mathrm{Ctrl}+\mathrm{O}\end{array}$}} \\
\hline & Export & $\rightarrow$ & & \\
\hline & Print & $C t r i+P$ & & \\
\hline & Close & Ctrl + W & & \\
\hline
\end{tabular}

Рисунок 7 - Загрузка FIS в рабочую область

и предыдущие 3 дня. Формат вызова функции имеет вид:

$$
\text { Out }=\text { evalfis }\left(\left[\begin{array}{lll}
0.13 & 0.110 .10 .098], ~ H D D 1)
\end{array}\right.\right.
$$

где out - имя выходной переменной; HDD1 - имя структуры FIS, которая была предварительно загружена в рабочую область системы MATLAB (рисунок 7).

Для загрузки структуры FIS в рабочую область MATLAB, нужно указать имя сгенерированной структуры (рисунок 8).

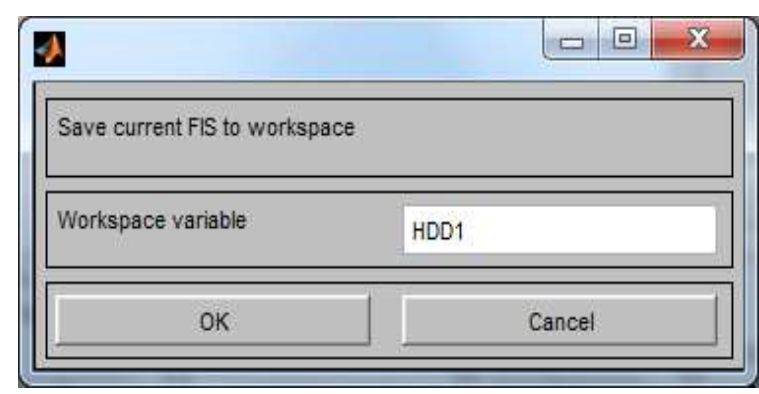

Рисунок 8 - Окно загрузки структуры FIS

Полученный результат команды evalfis представлен на рисунке 9.

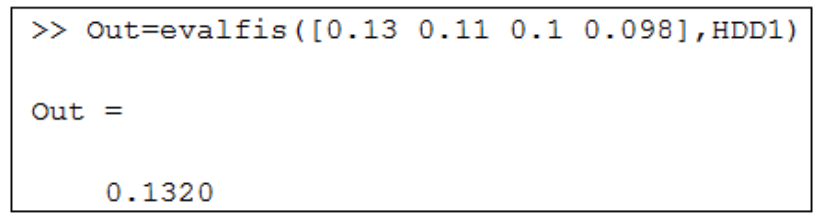

Рисунок 9 - Окно команд с отображением результатов оченки результата построенной нечеткой модели гибридной сети 
На рисунке видно, что полученный результат будет равен 0.1320 , что полностью совпадает со значением, указанным в обучающем файле, сгенерированным на основе графических статистических данных. Из этого можно сделать вывод, что построенная модель (нейро-нечеткая сеть) является применимой для прогнозирования выхода из строя жесткого диска сервера. Но, в отличие от вероятностной, она учитывает нелинейные зависимости и способна к самообучению. Таким образом, системный администратор, обладая полученной информацией, может самостоятельно произвести корректировку таблицы маршрутизации. Также адаптацию можно реализовать и программно.

\section{III. КЛАССИЧЕСКИЙ ПОДХОД К РЕШЕ- НИЮ ВОПРОСА ПОИСКА ОПТИМАЛЬНО- ГО МАРШРУТА. МАТРИЧНЫЙ АЛГОРИТМ}

Рассмотрим классический алгоритм поиска кратчайших путей в корпоративной сети. В качестве примера рассмотрим матричный алгоритм поиска наиболее оптимального пути от узла к узлу (типовой подход). В качестве исходных данных, к примеру, учитываются значения, характеризующие временную задержку между узлами сети, измеряемые в миллисекундах (мс).

Допустим, есть компьютерная сеть, имеющая вид графа, изображенного на рисунке 10 и матрица $L$, отображающая числовые значения непосредственных связей данной сети (1).

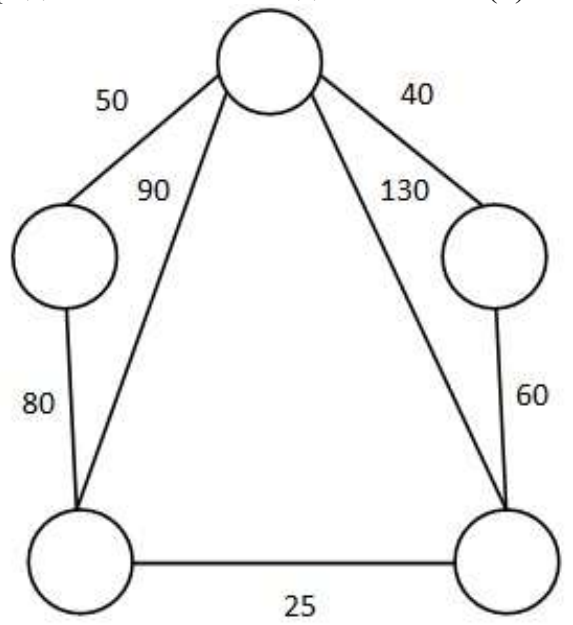
графа

Рисунок 10 - Компьютерная сеть в виде

$$
L=\left(\begin{array}{ccccc}
0 & 50 & \infty & 80 & \infty \\
50 & 0 & 40 & 90 & 130 \\
\infty & 40 & 0 & \infty & 60 \\
80 & 90 & \infty & 0 & 25 \\
\infty & 130 & 60 & 25 & 0
\end{array}\right)
$$

Возведение матрицы $L$ в степень максимального ранга $R_{\text {max }}$ даст нам матрицу оптимальных путей от узла к узлу между всеми парами узлов графа, матрицу оптимальных путей $L_{\text {опm }}=$ $L^{(\text {Rmax })}$

Если же при возведении матрицы $L$ в некоторую степень $q$ окажется, что:

$$
L^{q}=L^{(q-1)}
$$

то вычислительный процесс следует прервать, так как тождество (2) влечет за собой тождество (3).

$$
L^{q}=L^{(q+1)}
$$

Итак, вычислим для начала $L^{(2)}(4)-(6)$.

$$
\begin{aligned}
& l_{12}^{(2)}=\min \left(l_{11}^{(1)}+l_{12}^{(1)}, l_{12}^{(1)}+l_{22}^{(1)}, l_{13}^{(1)}+l_{32}^{(1)}, l_{14}^{(1)}+\right. \\
& \left.+l_{42}^{(1)}, l_{15}^{(1)}+l_{52}^{(1)}\right)=\min (50,50, \infty, 170, \infty)=50 \\
& l_{13}^{(2)}=\min \left(l_{11}^{(1)}+l_{13}^{(1)}, l_{12}^{(1)}+l_{23}^{(1)}, l_{13}^{(1)}+l_{33}^{(1)}, l_{14}^{(1)}+\right. \\
& \left.+l_{43}^{(1)}, l_{15}^{(1)}+l_{53}^{(1)}\right)=\min (\infty, 90, \infty, \infty, \infty)=90 \\
& l_{14}^{(2)}=\min \left(l_{11}^{(1)}+l_{14}^{(1)}, l_{12}^{(1)}+l_{24}^{(1)}, l_{13}^{(1)}+l_{34}^{(1)}, l_{14}^{(1)}+\right. \\
& \left.+l_{44}^{(1)}, l_{15}^{(1)}+l_{54}^{(1)}\right)=\min (80,140, \infty, 80, \infty)=80
\end{aligned}
$$

Аналогично рассчитав все остальные элементы матрицы $L^{(2)}$, получим следующую матрицу (7).

$$
L^{(2)}=\left(\begin{array}{ccccc}
0 & 50 & 90 & 80 & 105 \\
50 & 0 & 40 & 90 & 130 \\
90 & 40 & 0 & 85 & 60 \\
80 & 90 & 85 & 0 & 25 \\
105 & 130 & 60 & 25 & 0
\end{array}\right)
$$

Теперь сверяем полученную матрицу с матрицей исходной, и получаем $L^{(2)} \neq L$. Это означает, что поиск оптимальных путей следует продолжить. Для этого вычисляем $L^{(3)}=L^{(2)} * L(8)-(10)$.

$$
\begin{aligned}
& l_{12}^{(3)}=\min \left(l_{11}^{(2)}+l_{12}^{(1)}, l_{12}^{(2)}+l_{22}^{(1)}, l_{13}^{(2)}+l_{32}^{(1)}, l_{14}^{(2)}+\right. \\
& \left.+l_{42}^{(1)}, l_{15}^{(2)}+l_{52}^{(1)}\right)=\min (50,50,130,170,235)=50 \\
& l_{13}^{(3)}=\min \left(l_{11}^{(2)}+l_{13}^{(1)}, l_{12}^{(2)}+l_{23}^{(1)}, l_{13}^{(2)}+l_{33}^{(1)}, l_{14}^{(2)}+\right. \\
& \left.+l_{43}^{(1)}, l_{15}^{(2)}+l_{53}^{(1)}\right)=\min (\infty, 90,130,170,165)=90 \\
& l_{14}^{(3)}=\min \left(l_{11}^{(2)}+l_{14}^{(1)}, l_{12}^{(2)}+l_{24}^{(1)}, l_{13}^{(2)}+l_{34}^{(1)}, l_{14}^{(2)}+\right. \\
& \left.+l_{44}^{(1)}, l_{15}^{(2)}+l_{54}^{(1)}\right)=\min (80,140, \infty, 80,130)=80
\end{aligned}
$$

По аналогии рассчитываем и остальные элементы матрицы $L^{(2)}$. Полученная матрица $L^{(3)}$ будет иметь вид (11):

$$
L^{(3)}=\left(\begin{array}{ccccc}
0 & 50 & 90 & 80 & 105 \\
50 & 0 & 40 & 90 & 130 \\
90 & 40 & 0 & 85 & 60 \\
80 & 90 & 85 & 0 & 25 \\
105 & 130 & 60 & 25 & 0
\end{array}\right)
$$

Теперь воспользуемся формулой (2) и получим $L^{(3)}=L^{(2)}$. Из этого делаем вывод, что $L_{\text {опm }}=$ $L^{(3)}=L^{(2)}$, следовательно, оптимальные пути найдены, и расчёт матрицы 4 ранга можно не производить [6]. 
Итак, мы рассмотрели классический подход в вычислении оптимальных путей от узла к узлу в корпоративной сети. Данный метод является очень трудоёмким процессов, требующим огромного внимания и времени. Если корпоративная сеть будет иметь не 5 узлов, как в нашем частном случае, а 10 и более, то процесс расчета может сильно затянуться. При перепланировке сети, введении нового элемента или же при изменении длины линии связи, придётся пересчитывать всё сначала, на что понадобится много времени.

Предлагаемый алгоритм, который будет рассмотрен далее, основан на аппарате нейронечеткой логики [2, 8] и устраняет недостатки классического подхода. Помимо этого, данный алгоритм учитывает мнение и опыт эксперта сетевого администратора который знает о возможных задержках и других изменениях в параметрах КС. Опыт эксперта используется при создании функций принадлежности нейро-нечеткой или гибридной сети. При этом сеть реализует алгоритма Сугено [8].

Предположим, что имеется новая информация, каким образом изменяются параметры линии связи между узлами корпоративной сети в определенный момент времени. Данная информация представлена в виде матриц состояний и имеет вид (12) и (13).

$$
\begin{gathered}
M^{(1)}=\left(\begin{array}{ccccc}
0 & 50 & 90 & 80 & 105 \\
50 & 0 & 40 & 90 & 130 \\
90 & 40 & 0 & 85 & 60 \\
80 & 90 & 85 & 0 & 25 \\
105 & 130 & 60 & 25 & 0 \\
0 & 50 & 90 & 60 & 85 \\
50 & 0 & 40 & 90 & 130 \\
90 & 40 & 0 & 85 & 60 \\
60 & 90 & 85 & 0 & 25 \\
85 & 130 & 60 & 25 & 0
\end{array}\right)
\end{gathered}
$$

Предлагаемая интеллектуальная система, основанная на нейро-нечетком подходе [2, 7] будет прогнозировать изменение задержки на линии связи в определенный момент времени.

Для построения нейро-нечеткой системы прогноза, воспользуемся пакетом FuzzyTECH среды Matlab. C помощью графического редактора Anfis (вызывается из командной строки командой anfisedit) построим и обучим нашу систему. Для этого создадим файл с расширением *.dat, куда загрузим обучающие данные. После обучения системы, мы можем спрогнозировать изменения связей между узлами сети и сверить эти изменения с реальными значениями. Правила обучения будут иметь вид (рисунок 11).

Для проверки правильности полученных данных, спрогнозируем параметр линии связи с помощью аппарата нейро-нечеткой логики. Ре- зультат работы программы представлен на рисунке 12.

На результате видно полное сходство полученных результатов и результатов из выражения (11). Этот подход позволяет адаптировать маршрутизатор с учетом прогнозной задержки между узлами.

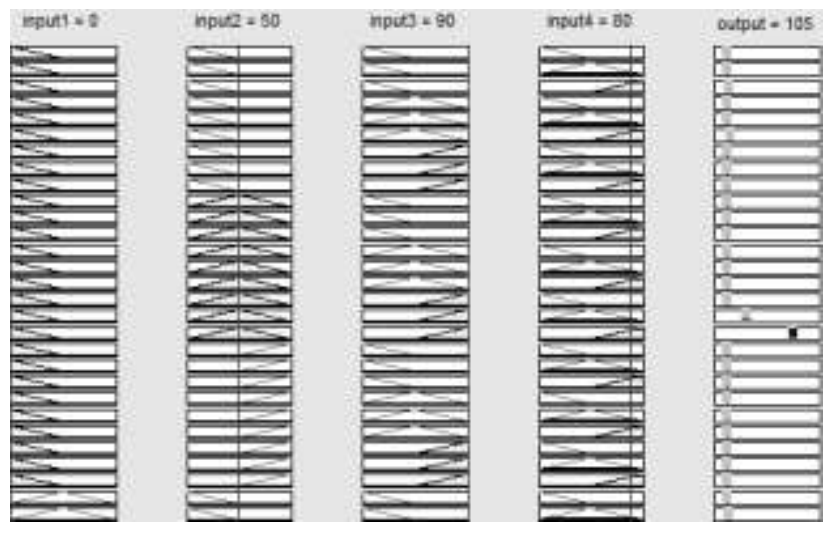

Рисунок 11 - Диалоговое окно правил обучения нейро-нечеткой сети

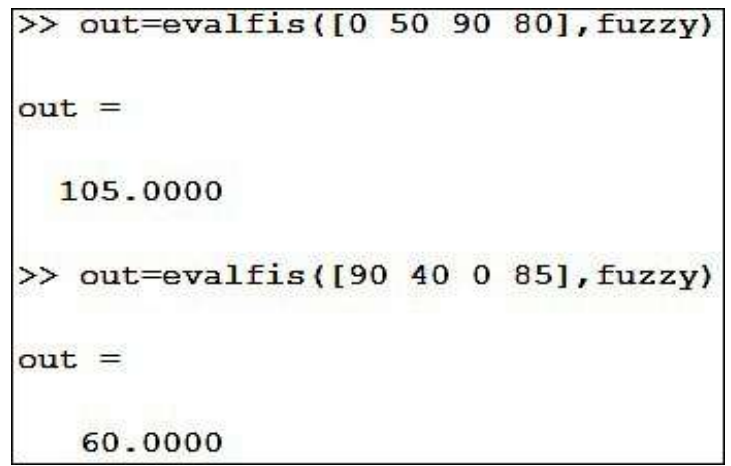

Рисунок 12 - Результаты прогнозирования

\section{ЗАКЛЮЧЕНИЕ}

Перспективным подходом в решении проблем эффективной работы КС на основе успешной маршрутизации является интеллектуальный адаптивный подход.

Проверка построенной нейро-нечеткой модели показывает высокую степень ее адекватности, что даёт нам возможность применять данную модель для прогнозирования выхода из строя жестких дисков и другого оборудования КС и учитывать эту информацию в дальнейшем для адаптации маршрутизатора.

Нейро-нечёткий подход, по мнению авторов, является перспективным, так как интеллектуальные системы учитывают мнение экспертов, способны самообучаться и работать с нелинейными данными.

При прогнозировании временных задержек информации на линии связи между узлами сети, можно заметить абсолютное сходство прогнозных 
результатов с исходными. Это даёт возможность применять данную модель на практике и поможет решить проблему поиска оптимального маршрута между узлами сети в кротчайшие сроки с учетом двух прогнозных параметров (надежности и задержки) по сравнению с классическими алгоритмами.

\section{ЛИТЕРАТУРА}

1. Білуха М.Т. Основи наукових досліджень [Текст] / К. : Вища школа, 1997. - 271 с.

2. Бодянский Е.В., Кучеренко Е.И., Михалев А.И. Нейро-фаззи сети в задачах моделирования сложных систем [Текст] / Монография (научное издание), Днепропетровск, «Системный технолог», 2005. - $311 \mathrm{c}$.

3. Воронин А. А., Морозов Б. И. Надежность информационных систем [Текст] / СанктПетербург, «СПбГТУ», 2001. - 89 с.

4. Гольдштейн Б. С. - Протоколы сети доступа. Том 2 [Текст] / Москва, «Радио и связь», 1999. $313 \mathrm{c}$.

5. Гольдштейн Б.С., Пинчук А.В., Суховицкий А.Л. ІР-телефония [Текст] / Москва, «Радио и связь», 2001. - 137 с

6. Князева Н.А. - Теория проектирования компьютерных систем и сетей [Текст] / Обучающее пособие для ВУЗов, 2012, 239 с.

7. Леинванд А., Пински Б. Конфигурирование маршрутизаторов Cisco, 2-е издание [Текст] / Мocква, «Вильямс», 2001, 368 с

8. Леоненко А. В. - Нечеткое моделирование в среде MATLAB и FuzzyTECH [Текст] / СанктПетербург, «БХВ-Петербург», 2005. - 736 с.

9. Сырцев А. В., Тимофеев А. В. - Нейронная и мультиагентная маршрутизация в телекоммуникационных сетях [Текст] / Международный журнал «Теория и практическое применение», 2003. - 172 c.

10. Интернет-сайт http://habrahabr.ru. А. Ализар «Опубликована статистика поломок жестких дисков Google» // Електронный ресурс, Статья № 13560. - Режим доступа: http://habrahabr.ru/post/13560/. [27. 08. 2007]

11. Tanenbaum A., Wetherall D. - Computer Networks, 5th edition. / «Prentice Hall», 962 p.

12. Интернет-сайт dissercat.com - Электронная библиотека диссертаций и авторефератов. Д. А. Перепёлкин «Методы и алгоритмы адаптивной маршрутизации в корпоративных вычислительных сетях» // Электронный ресурс. Режим доступа: http://www.dissercat.com/content/metody-i-algoritmyadaptivnoi-marshrutizatsii-v-korporativnykh-

vychislitelnykh-setyakh. [2009]

13. Интернет-сайт http://sci-library.com/ - Библиотека диссертаций и авторефератов защищенных в России. А. М. Комиссаров «Адаптивная маршрутизация в сетях передачи данных с учетом самоподобия трафика» // Электронный ресурс. - Режим доступа: http://sci-library.com/thesis/adaptivnaya- marshrutizatsiya-v-setyah-peredachi-dannyh-s-uchetom-samopodobiya-trafika [2011]

14. Интернет-сайт dissercat.com - Электронная библиотека диссертаций и авторефератов. М. П. Березка «Методы и модели адаптивной маршрутизации в сетях ЭВМ «Экспресс-2» // Электронный peсурс. - Режим доступа:

http://www.dissercat.com/content/metody-i-modeliadaptivnoi-marshrutizatsii-v-setyakh-evm-na-primereseti-evm-ekspress-2. [2001]

\section{REFERENCES}

1. Biluha M. T. Osnovy naukovykh doslidzhen Kiev. : Vysshaya shkola, 1997. - 271 p. (in Ukrainian) 2. Bodjanskii E. V., Kucherenko E. I., Mihalev A. I. Neiro-Fuzzy seti $\mathrm{v}$ zadachah modelirovaniya slozhnyh sistem / Dnepropetrovsk : Sistemnyi tehnolog, 2005. - 311 p. (in Russian).

3. Voronin A.A., Morozov B.I. Nadezhnost informatsionnyh sistem / St.-Petersburg : 2001. - 89 p. (in Russian)

4. Goldshtein B. S. Protokoly seti dostupa. Tom $2-$ Moscow: Radio i Kommunikatsyi, 1999. - 313 p. [in Russian].

5. Goldshtein B. S., Pinchuk A. V., Suhovitskij A. L. IP telefoniya - Moscow, Radio and Communication, 2001. - 137 p. (in Russian)

6. Knyazeva N.A. Teoriya proektirovaniya kompjuternyh sistem / Odessa: - Scientific Guide for Universities, 2012, 239 p. (in Ukrainian)

7. Leinvand A., Pinsky B. Konfigurirovanie marshrutizatorov Cisco. 2-e izdanie / Moskow, Viliams, 2001, 368 p. (in Russian)

8. Leonenko A. V. - Nechetkoe modelirovanie $\mathrm{v}$ srede Matlab I FuzzyTech - St. Petersburg, BHVPeterburg, 2005. - 736 p. (in Russian)

9. Syrtzev A. V., Timofeev A. V. Neironnaya I multiagentnaya marshrutizatsiya $\mathrm{v}$ telekommunikatsionnyh setjah // International Journal "Information Theories and Their Applications". 2003. Vol.10, no. 2.P. 167-172. (in Russian)

10. Web site http://habrahabr.ru [Electronic resource] Paper № 13560. - Mode of access to information: http://habrahabr.ru/post/13560/ [27. 08. 2007]. (in Russian)

11. Tanenbaum A.D. Wetherall Computer Networks, 5th edition [Text]. / «Prentice Hall», 962 p. (in English)

12. Web site dissercat.com - Electronic Library of dissertations and theses. D. A. Perepjolkin «Metody i algoritmy adaptivnoi marshrutizatsii $\mathrm{v}$ korporativnyh setjah» // electronic resource. - access mode: http://www.dissercat.com/content/metody-i-algoritmyadaptivnoi-marshrutizatsii-v-korporativnykh-

vychislitelnykh-setyakh. [2009]

13. Web site http://sci-library.com/ - Library dissertations defended in Russia. A. M. Komissarov «Adaptivnaja marshrutizatsija $\mathrm{V}$ setjah peredachi dannyh s uchetom samopodobija trafika» // electronic resource. - Access mode: http://sci-library.com /thesis/adaptivnaya-marshrutizatsiya-v-setyah- 
peredachi-dannyh-s-uchetom-samopodobiya-trafika [2011]

14. Web site dissercat.com - Electronic Library of dissertations and theses. M. P. Berezka «Metody i modeli adaptivnoj marshrutizatsii $\mathrm{v}$ kompjuternyh setjah "Ekspress-2"»// / electronic resource. - Access mode:

http://www.dissercat.com/content/metody-i-modeliadaptivnoi-marshrutizatsii-v-setyakh-evm-na-primereseti-evm-ekspress-2. [2001]

\section{APPLICATION OF NEURO-FUZZY APPROACH TO IMPROVE RELIABILITY AND OPTIMAL WORK OF COMPUTER NETWORK}

This article focuses on the application of neuro-fuzzy approach to improve the reliability of the optimal network. This work is divided into two parts. In the first part, the author, using a program written in Matlab, predicts the probability of failure of the hard disk. For this purpose, the statistics, which presented on the web-page habrahabr.ru by Corporation Google Inc was used. Using these data, the author has taught previously created a hybrid neural network training method with an error rate of zero. Validation of the developed model was performed using the command from the command line evalfis editor Matlab. The result completely coincided with the original statistical value. This indicates that the model can be successfully used in practice. At the end of the first part of the author of the specified benefits of neuro-fuzzy approach for the task, namely, accounting expert opinions and the ability to self-learning neural network. The second part of the article is devoted to the routing in computer networks. Classical matrix approach for solving the problem of finding the optimal route from node to node in a computer network was considered. As an alternative to the classical approach, an algorithm based on neuro-fuzzy logic was considered. The proposed intelligent system, which based on neuro-fuzzy approach, will predict the delay variation on a link at a time. To build this system package FuzzyTech environment Matlab was used. When forecasting the time delay information on the communication line between the nodes of the network, you will notice the absolute similarity with the original forecast results. This makes it possible to apply this model in practice and help solve the problem of finding the optimal route between network nodes in the shortest possible time taking into account the two predicted parameters (delay and reliability) compared to classical algorithms.

Keywords: neuro-fuzzy network, adaptive routing, intelligent prediction, neural network, training of the network, matrix algorithm, self-learning network, learning rules, activation function, hybrid network, optimal route.

Отримана в редакції 16.01.2014, прийнята до друку 04.03.2014 